Draft VERSION SEPTEMBER 11, 2018

Preprint typeset using $\mathrm{AT}_{\mathrm{E} X}$ style emulateapj v. 11/10/09

\title{
THE LICK-CARNEGIE SURVEY: FOUR NEW EXOPLANET CANDIDATES
}

\author{
Stefano Meschiari $^{1}$, Gregory Laughlin ${ }^{1}$, Steven S. Vogt ${ }^{1}$, R. Paul Butler ${ }^{2}$, Eugenio J. Rivera ${ }^{1}$, Nader \\ HAGHighipour $^{3}$ AND PETER JALOWICZOR \\ Draft version September 11, 2018
}

\begin{abstract}
We present new precise HIRES radial velocity (RV) data sets of five nearby stars obtained at Keck Observatory. HD 31253, HD 218566, HD 177830, HD 99492 and HD 74156 are host stars of spectral classes $\mathrm{F}$ through $\mathrm{K}$ and show radial velocity variations consistent with new or additional planetary companions in Keplerian motion. The orbital parameters of the candidate planets in the five planetary systems span minimum masses of $\mathcal{M} \sin i=27.43 \mathcal{M}_{\oplus}$ to $8.28 \mathcal{M}_{J}$, periods of 17.05 to 4696.95 days and eccentricities ranging from circular to extremely eccentric $(e \approx 0.63)$.

The 5th star, HD 74156, was known to have both a 52-day and a 2500-day planet, and was claimed to also harbor a 3rd planet at 336d, in apparent support of the "Packed Planetary System" hypothesis. Our greatly expanded data set for HD 74156 provides strong confirmation of both the 52-day and 2500-d planets, but strongly contradicts the existence of a 336-day planet, and offers no significant evidence for any other planets in the system.
\end{abstract}

Subject headings: Planets and satellites: detection, Methods: numerical

\section{INTRODUCTION}

The planetary census has reached an impressive 496 extrasolar planets. Planetary companions have been successfully detected using a variety of techniques, primarily radial velocities (463; see e.g. Mavor \& Queloz 1995; Butler et al. 2006; Udry et al. 2007) and transit photometry (106; see e.g. Henry et al. 2000; Charbonneau et al. 2000, 2007). Other techniques employed include microlensing (Bennett et al. 2009), astrometry (Benedict et al. 2002; Bean \& Seifahrt 2009), stellar pulsations (Silvotti et al. 2007) and even direct imaging (Chauvin et al. 2005; Kalas et al. 2008; Marois et al. 2008) 4

The radial velocity method has been used to either detect or characterize more than $90 \%$ of all currently known planets, and continues to be a very important technique. Both its continued productivity (e.g. Valenti \& Fischer 2005) and its ability to accurately probe planetary architectures into the vicinity of the terrestrial mass region (e.g Rivera et al. 2005; Mayor et al. 2009; Vogt et al. 2010) are a testament to the rapid technological advances.

We have been monitoring a large set of nearby stars under precise radial velocity survey with the High Resolution Echelle Spectrometer (HIRES) at Keck for the past 17 years. In this paper, we present new radial velocity (RV) observations for five of our target stars: HD 31253, HD 218566, HD 177830, HD 99492 and HD 74156.

The plan of this paper is as follows. In Section 2 we discuss the procedure followed to obtain and reduce the RV dataset. In Sections 3 through 7 we describe the

\footnotetext{
${ }^{1} \mathrm{UCO} /$ Lick Observatory, Department of Astronomy and Astrophysics, University of California at Santa Cruz, Santa Cruz, CA 95064

${ }^{2}$ Department of Terrestrial Magnetism, Carnegie Institute of Washington, Washington, DC 20015

${ }^{3}$ Institute for Astronomy and NASA Astrobiology Institute, University of Hawaii-Manoa, Honolulu, HI 96822

4 Source: http://www.exoplanet.eu, retrieved on 06/14/2010
}

main stellar properties, derive model Keplerian fits with associated parameter uncertainties and discuss the planetary systems they imply. Finally, we discuss the new planetary companions in Section 8

\section{RADIAL VELOCITY OBSERVATIONS AND TARGET STARS}

The HIRES spectrometer (Vogt et al. 1994) of the Keck-I telescope was used for all the new RVs presented in this paper. Doppler shifts were measured in the usual manner (Butler et al. 1996) by placing an Iodine absorption cell just ahead of the spectrometer slit in the converging beam from the telescope. This gaseous Iodine absorption cell superimposes a rich forest of Iodine lines on the stellar spectrum, providing a wavelength calibration and proxy for the point spread function (PSF) of the spectrometer. The Iodine cell is sealed and temperaturecontrolled to $50 \pm 0.1 \mathrm{C}$ such that the column density of Iodine remains constant. For the Keck planet search program, we operate the HIRES spectrometer at a spectral resolving power $\mathrm{R} \approx 70,000$ and wavelength range of $3700-8000 \AA$, though only the region 5000-6200 $\AA$ (with Iodine lines) is used in the present Doppler analysis. A block of the spectrum containing the Iodine region is divided into $\sim 700$ chunks of $2 \AA$ each. Each chunk produces an independent measure of the wavelength, PSF, and Doppler shift. The final measured velocity is the weighted mean of the velocities of the individual chunks. All radial velocities have been corrected to the solar system barycenter, but are not tied to any absolute radial velocity system. As such, they are "relative" radial velocities, with a zero point that is usually set simply to the mean of each set.

The internal uncertainties quoted for all the RV's in this paper reflect only one term in the overall error budget, and results from a host of systematic errors from characterizing and determining the PSF, detector imperfections, optical aberrations, effects of under-sampling the Iodine lines, etc. Two additional major sources of error are photon statistics and stellar jitter. The latter 
TABLE 1

STELLAR PARAMETERS

\begin{tabular}{|c|c|c|c|c|c|}
\hline Parameter & HD 31253 & HD 218566 & HD 177830 & HD 99492 & HD 74156 \\
\hline Spec. Type & F8 & $\mathrm{K} 3 \mathrm{~V}$ & KOIV & $\mathrm{K} 2 \mathrm{~V}$ & G1V \\
\hline$M_{v}$ & $3.48^{\mathrm{a}}$ & $6.21^{\mathrm{b}}$ & 3.3 & 6.3 & $3.56^{\mathrm{c}}$ \\
\hline$B-V$ & 0.58 & 1.013 & 1.091 & 1.053 & 0.581 \\
\hline$V$ & 7.133 & 8.628 & 7.177 & 7.383 & 7.614 \\
\hline $\operatorname{Mass}\left(\mathcal{M}_{\odot}\right)$ & $1.23[0.05]$ & $0.85[0.03]^{\mathrm{d}}$ & 1.47 & $0.83^{\mathrm{d}}$ & 1.24 \\
\hline Radius $\left(\mathcal{R}_{\odot}\right)$ & $1.71[0.17]$ & $0.86[0.08]$ & $2.62[0.06]$ & $0.96[0.11]$ & $1.64[0.19]$ \\
\hline Luminosity $\left(L_{\odot}\right)$ & $3.286[0.446]$ & $0.353[0.032]$ & $4.842[1.003]$ & $0.418[0.057]$ & $3.037[0.485]$ \\
\hline Distance (pc) & $53.82[3.45]$ & $29.94[1.11]$ & $59.03[2.77]^{]}$ & $17.99[1.14]$ & $64.56[4.93]$ \\
\hline$V \sin i\left(\mathrm{~km} \mathrm{~s}^{-1}\right)$ & 3.8 & 0.0 & 2.5 & 1.4 & 4.3 \\
\hline$S_{h k}$ & $0.141[0.018]$ & 0.297 & $0.125[0.016]$ & $0.254[0.033]$ & 0.144 \\
\hline $\log R_{h k}$ & -5.11 & -4.88 & -5.37 & -4.84 & -5.08 \\
\hline Age (Gyr) & 3 & 8.5 & $2.2-6.6$ & 4 & $3.7[0.4] \mathrm{d}$ \\
\hline$[F e / H]$ & 0.16 & 0.38 & $0.545[0.03]$ & 0.36 & 0.13 \\
\hline$T_{e f f}(K)$ & 5960.0 & 4820.0 & 4949 & 4740.0 & $5960.0[100.0]$ \\
\hline $\log g$ & 4.1 & 4.81 & 3.65 & 4.77 & $4.4[0.15]^{\mathrm{c}}$ \\
\hline$P_{\text {rot }}$ & 23 & - & $65^{\mathrm{f}}$ & $45^{\mathrm{e}}$ & - \\
\hline
\end{tabular}

a Nordström et al. (2004)

b Wright et al. (2004)

c Naef et al. (2004)

d Takeda et al. (2007)

e Marcy et al. (2005)

${ }^{\mathrm{f}}$ Barnes (2001)

TABLE 2

KEPLERIAN ORBITAL SOLUTIONS

\begin{tabular}{|c|c|c|c|c|c|}
\hline Parameter & HD 31253 & HD 218566 & HD 177830 & HD 99492 & HD 74156 \\
\hline$P(\mathrm{~d})$ & $466[3] \dagger$ & $225.7[0.4] \dagger$ & $\begin{array}{c}406.6[0.4] \\
110.9[0.1] \dagger\end{array}$ & $\begin{array}{c}17.054[0.003] \\
4970[744] \dagger\end{array}$ & $\begin{array}{c}2520[15] \\
51.638[0.004]\end{array}$ \\
\hline$e$ & $0.3[0.2]$ & $0.3[0.1]$ & $\begin{array}{c}0.009[0.004] \\
0.3[0.1]\end{array}$ & $\begin{array}{r}0.13[0.07] \\
0.1[0.2]\end{array}$ & $\begin{array}{l}0.38[0.02] \\
0.63[0.01]\end{array}$ \\
\hline$K\left(\mathrm{~m} \mathrm{~s}^{-1}\right)$ & $12[2]$ & $8.3[0.7]$ & $\begin{array}{c}31.6[0.6] \\
5.1[0.8]\end{array}$ & $\begin{array}{l}7.9[0.6] \\
4.9[0.7]\end{array}$ & $\begin{array}{l}115[3] \\
108[4]\end{array}$ \\
\hline$T_{\text {peri }}-2,440,000(\mathrm{JD})$ & $10660[19]$ & $10360[154]$ & $\begin{array}{c}10154[35] \\
10179[7]\end{array}$ & $\begin{array}{c}10449[2] \\
9636[2210]\end{array}$ & $\begin{array}{c}8416[33] \\
10793.3[0.2]\end{array}$ \\
\hline$\varpi(\operatorname{deg})$ & 244 [23] & $36[24]$ & $\begin{array}{c}85[31] \\
110[29]\end{array}$ & $\begin{array}{l}196[32] \\
38[64]\end{array}$ & $\begin{array}{l}268[4] \\
174[2]\end{array}$ \\
\hline$M \sin i\left(\mathcal{M}_{J}\right)$ & $0.50[0.07]$ & $0.21[0.02]$ & $\begin{array}{l}1.49[0.03] \\
0.15[0.02]\end{array}$ & $\begin{array}{r}0.087[0.006] \\
0.36[0.06]\end{array}$ & $\begin{array}{r}8.2[0.2] \\
1.78[0.04]\end{array}$ \\
\hline$a(\mathrm{AU})$ & $1.260[0.006]$ & $0.6873[0.0008]$ & $\begin{array}{l}1.2218[0.0008] \\
0.5137[0.0003]\end{array}$ & $\begin{array}{c}0.12186[0.00002] \\
5.4[0.5]\end{array}$ & $\begin{array}{c}3.90[0.02] \\
0.29169[0.00001]\end{array}$ \\
\hline$P_{t r}$ & 0.004 & 0.007 & $\begin{array}{l}0.004 \\
0.010\end{array}$ & $\begin{array}{l}0.04 \\
0.0008\end{array}$ & $\begin{array}{c}0.001 \\
0.03\end{array}$ \\
\hline$N_{\text {obs }}$ [Median uncertainty] & KECK: 39 [1.59] & KECK: 56 [1.27] & KECK: 88 [1.06] & KECK: 93 [1.36] & $\begin{array}{c}\text { CORALIE04: } 44[7] \\
\text { ELODIE04: } 51[12] \\
\text { HET09: } 82[8] \\
\text { KECK: } 29[1.98]\end{array}$ \\
\hline Epoch (JD) & 2450838.7519 & 2450366.8550 & 2450276.0239 & 2450462.1140 & 2450823.5570 \\
\hline$\chi^{2}$ & 8.87 & 8.41 & 15.31 & 7.17 & 3.09 \\
\hline $\operatorname{RMS}\left(\mathrm{m} \mathrm{s}^{-1}\right)$ & 4.23 & 3.48 & 3.85 & 3.22 & 12.80 \\
\hline Jitter $\left(\mathrm{m} \mathrm{s}^{-1}\right)$ & 3.92 & 3.23 & 3.71 & 2.94 & 8.59 \\
\hline
\end{tabular}

Note. - $†$ New planet candidate

varies widely from star to star, and can be mitigated to some degree by selecting magnetically-inactive older stars and by time-averaging over the star's unresolved low-degree surface p-modes. All observations have been further binned on 2-hour timescales.

We present in Table 1 a few basic parameters (and uncertainties, where available) for all the host stars considered in this paper. Unless otherwise noted, the data are mostly as listed in the SPOCS database Valenti \& Fischer 2005) and the NASA
NStED database.

Table 2 summarizes all the Keplerian fits for the target stars in this paper; they will be discussed in more detail in the following sections. The orbital fits were derived using the Systemic Console (Meschiari et al. 2009)6. The errors on each parameter are estimated using the bootstrap technique with 5000 scrambled realizations of the

5 http://nsted.ipac.caltech.edu/

6 Downloadable at http://www.oklo.org 
RV datasets. For each planet, we list best-fit period $(P)$, eccentricity $(e)$, semi-amplitude $(K)$, time of periastron passage $\left(T_{\text {peri }}\right)$, longitude of pericenter $(\varpi)$, minimum $\operatorname{mass}(\mathcal{M} \sin i)$ and semi-major axis $(a)$. Additionally, we report approximate estimates of the transit probability calculated as part of the Monte-Carlo modeling, assuming a putative radius $\mathcal{R}=\mathcal{R}_{J U P}$.

\section{HD 31253 (HIP 22826) \\ 3.1. Stellar properties}

HD 31253 is a $\mathrm{V}=7.133$ magnitude star of spectral class F8. Relative to the Sun, HD 31253 is modestly metal-rich $([\mathrm{Fe} / \mathrm{H}]=0.16)$.

This $1.23 \mathcal{M} \odot$ star has a reported $V \sin i$ of $3.8 \mathrm{~m} \mathrm{~s}^{-1}$ which, in conjunction with its derived radius, implies a maximum rotation period of about 23 days. Our measurement of $\log R_{h k}^{\prime}=-5.13$ agrees well with that listed on the NASA NStED site, and leads to an estimate of $\sim 2.3$ for the expected radial velocity jitter due to stellar surface activity (Wright 2005).

\subsection{Keplerian solution}

Table 3 shows the complete set of 39 relative radial velocity observations for HD 31253. The radial velocity coverage spans approximately 13 years of RV monitoring. The median internal uncertainty for our observations is $1.59 \mathrm{~m} \mathrm{~s}^{-1}$, and the peak-to-peak velocity variation is $36.37 \mathrm{~m} \mathrm{~s}^{-1}$. The velocity scatter around the average $\mathrm{RV}$ in our measurements is $8.92 \mathrm{~m} \mathrm{~s}^{-1}$.

The top panel of Figure 1 shows the individual RV observations for HD 31253 . The middle panel shows the error-weighted Lomb-Scargle (LS) periodogram of the full RV dataset (Gilliland \& Baliunas 1987). The three horizontal lines in this figure and other comparable plots represent, from top to bottom, the $0.1 \%, 1.0 \%$, and $10.0 \%$ analytic False Alarm Probability (FAP) levels, respectively. The analytic FAPs are computed using a straightforward approach, where we estimate the number of independent frequencies by analyzing a set of 1,000 gaussian deviates with the same timestamps as the original dataset (Press et al. 1992).

For the highest peak, the quoted FAP is estimated using a more robust Monte Carlo approach, which consists of generating sets of scrambled realizations of the dataset and determining the maximum periodogram power for each (e.g. Marcy et al. 2005). For all the datasets presented in this paper, we analyze $3 \times 10^{5}$ scrambled datasets.

The computed FAP for the strong Keplerian signal at $P=460.32$ days in the RV dataset indicates an estimated FAP $\approx 4 \times 10^{-5}$. Finally, the lower panel of Figure 1 shows the spectral window. A peak at frequency, $f_{s}$ in the spectral window function can be associated with aliases occurring at $\left|f_{p} \pm f_{s}\right|$, where $f_{p}$ is a true periodicity of the input signal. For more details, see Dawson \& Fabrycky (2010). Peaks in the spectral window function are often associated with relatively immutable periodicities in the observational cadence, such as those arising from the sidereal and solar day, the lunar synodic month and the solar year. The strongest peak in the periodogram is well-fit by a Keplerian orbit of period 465.54 days and semi-amplitude $K=12.22$ $\mathrm{m} \mathrm{s}^{-1}$. Together with the assumed stellar mass of
TABLE 3

KECK RADIAL VELOCITIES FOR HD 31253 (Sample: full table in electronic version)

\begin{tabular}{ccc}
\hline \hline Barycentric JD & $\mathrm{RV}\left[\mathrm{m} \mathrm{s}^{-1}\right]$ & Uncertainty $\left[\mathrm{m} \mathrm{s}^{-1}\right]$ \\
\hline 2450838.75 & -1.44 & 1.97 \\
2451043.12 & -11.98 & 1.64 \\
2451073.03 & -10.12 & 1.43 \\
2451170.91 & 6.52 & 1.78 \\
2451228.79 & 11.64 & 1.38 \\
2451411.13 & 3.54 & 2.19 \\
2451550.87 & -20.13 & 1.58 \\
2451581.86 & -4.86 & 1.69 \\
2451757.13 & 7.45 & 1.58 \\
2451793.12 & 8.50 & 1.78 \\
2451883.00 & 2.05 & 1.80 \\
2451884.08 & -0.63 & 1.73 \\
2451898.01 & 6.44 & 1.61 \\
2451899.00 & -2.59 & 1.54 \\
2451899.99 & 1.53 & 1.48 \\
2451901.01 & -1.13 & 1.47 \\
2451973.75 & -7.57 & 1.83 \\
2451974.76 & -7.26 & 1.63 \\
2452235.85 & 1.28 & 1.62 \\
2452536.09 & 0.00 & 1.55
\end{tabular}

$1.23 \mathcal{M}_{\odot}$, this amplitude implies a minimum mass of $\mathcal{M} \sin i=0.50 \mathcal{M}_{J}$. The best-fit orbit for the planet is mildly eccentric $(e \approx 0.34)$. This 1 -planet fit achieves a reduced $\chi^{2}=8.87$, with an RMS of $4.23 \mathrm{~m} \mathrm{~s}^{-1}$. The expected jitter of HD 31253 (that is, the amount of jitter required to bring the reduced $\chi^{2}$ of the best-fit solution to 1.0 ) is $3.92 \mathrm{~m} \mathrm{~s}^{-1}$.

The top panel of Figure 2 shows the phased stellar reflex velocity of HD 31253 compared to the RV dataset. The middle panel shows the residuals to the 1-planet solution. Finally, the bottom panel shows the periodogram of the residuals of the best-fit solution. No interesting peaks are evident, indicating that the present data set provides no strong support for additional planets in the system.

We do not have photometry of HD 31253 that might conclusively rule out stellar rotation signatures as a cause of the RV variations. But this star does have a measured $V \sin i$ of $3.8 \mathrm{~m} \mathrm{~s}^{-1}$ which implies a maximum rotation period of about 23 days, much shorter than the 466-d Keplerian period. The semi-amplitude of the observed variations is $12 \mathrm{~m} \mathrm{~s}^{-1}$, whereas a 466 -d rotation period, combined with a stellar radius of $1.71 \mathcal{R}_{\odot}$, would not produce radial velocity effects above a few tenths of a $\mathrm{m}$ $\mathrm{s}^{-1}$. Therefore, stellar rotation can sensibly be ruled out as being responsible for the observed RV variations.

\section{HD 218566 (HIP 114322)}

\subsection{Stellar properties}

HD 218566 is a $\mathrm{V}=8.628$ magnitude star of spectral class K3V. In comparison to the Sun, HD 218566 is quite metal-rich $([\mathrm{Fe} / \mathrm{H}]=0.38)$. Table 1 reports some of the salient stellar properties, as reported by NStEd, Wright et al. (2004) and Takeda et al. (2007).

\subsection{Keplerian solution}

Table 4 shows the 56 relative radial velocity observations for HD 218566. The radial velocity coverage spans approximately 14 years of RV monitoring. The median internal uncertainty for our observations is $1.27 \mathrm{~m} \mathrm{~s}^{-1}$, 

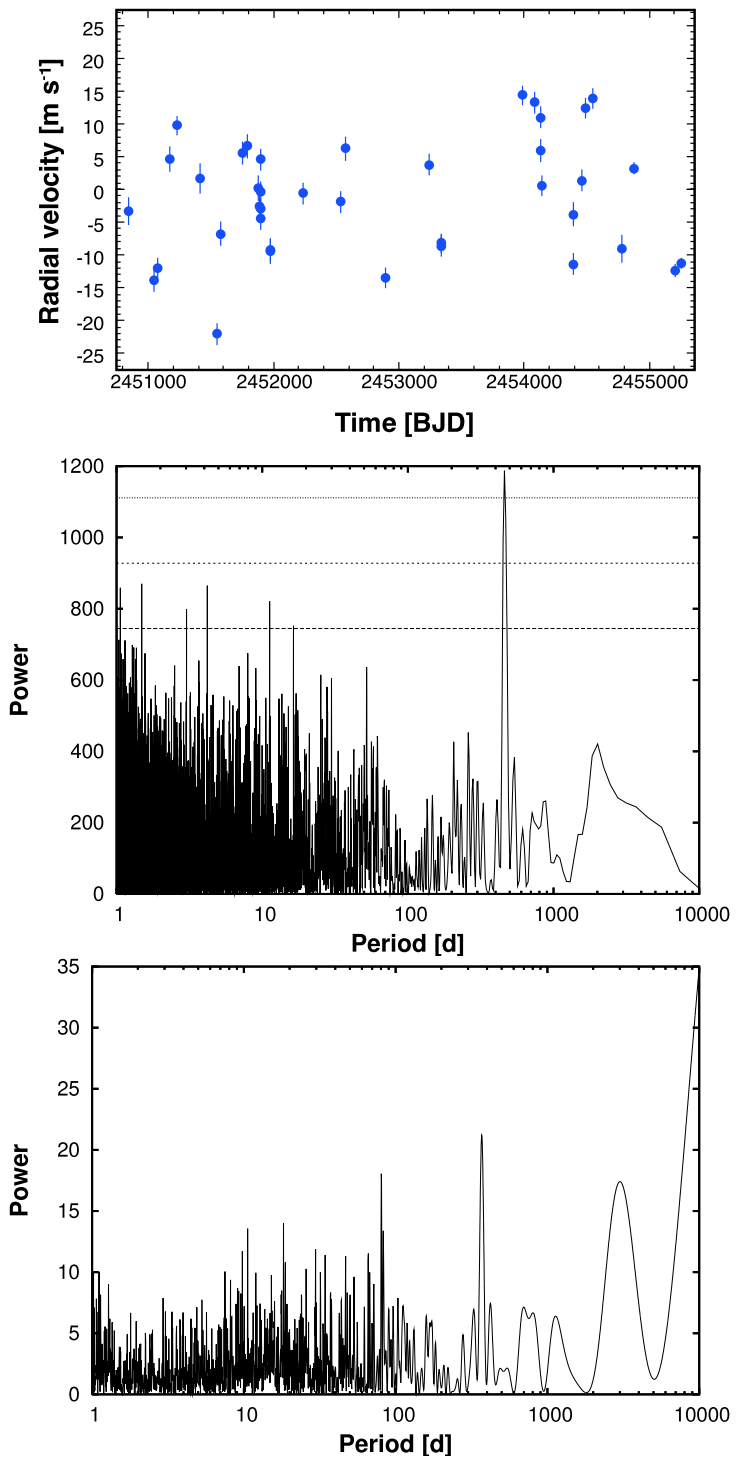

Fig. 1.- Radial velocity data and periodograms for HD 31253. Top panel: Relative radial velocity data obtained by KECK. Middle panel: Error-weighted Lomb-Scargle periodogram of the radial velocity data. Bottom panel: Power spectral window.

and the peak-to-peak velocity variation is $28.46 \mathrm{~m} \mathrm{~s}^{-1}$. The velocity scatter around the mean RV in our measurements is $7.18 \mathrm{~m} \mathrm{~s}^{-1}$.

The top panel of Figure 3 shows the individual RV observations for HD 218566. The middle panel shows the error-weighted Lomb-Scargle (LS) periodogram of the full RV data set, while the bottom figure shows the spectral window. The FAP calculation for the strong Keplerian signal at $P=225.06$ days in the RV dataset indicates an estimated FAP $\approx<4 \times 10^{-6}$. The dominant peak in the periodogram can be explained by a Keplerian orbit of period 225.73 days and semi-amplitude $K=8.34 \mathrm{~m} \mathrm{~s}^{-1}$. This amplitude suggests a minimum mass of $\mathcal{M} \sin i=0.21 \mathcal{M}_{J}$ (assuming a stellar mass of $\left.0.88 \mathcal{M}_{\odot}\right)$. The best-fit orbit for the planet is moderately eccentric $(e \approx 0.37)$. This fit achieves a reduced $\chi^{2}=8.41$, with an RMS of $3.48 \mathrm{~m} \mathrm{~s}^{-1}$. The expected jitter of HD 218566 (that is, the amount of jitter required to bring the reduced $\chi^{2}$ of the best-fit solution to 1.0) is
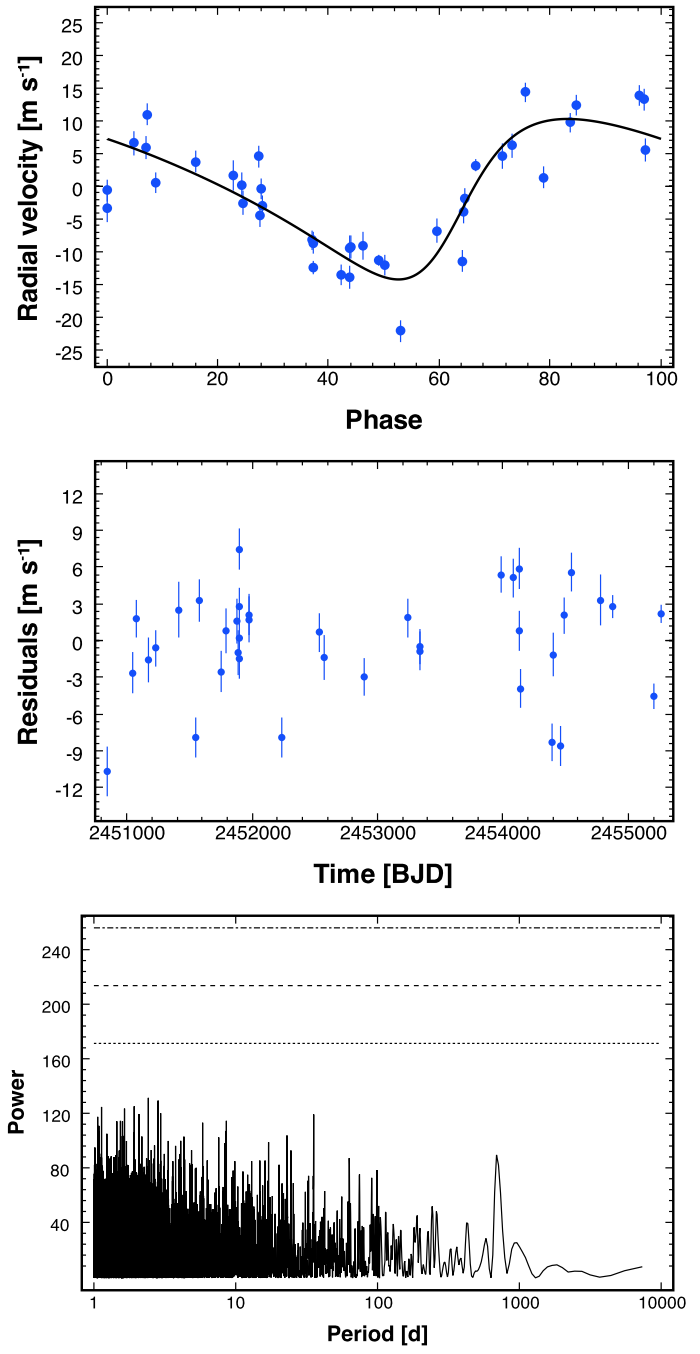

FIG. 2.- Keplerian solution and residuals periodogram for HD 31253. Top panel: Phased Keplerian fit. Middle panel: Residuals to the 1-planet Keplerian fit. Bottom panel: Periodogram of the residuals to the 1 -planet best-fit solution.

\section{$3.23 \mathrm{~m} \mathrm{~s}^{-1}$.}

The top panel of Figure 4 shows the phased stellar reflex velocity of HD 218566 compared to the RV dataset. The middle panel shows the residuals to the 1-planet solution. The periodogram of the residuals to the best-fit solution, shown in the bottom panel, displays no strong peaks that would support the evidence for additional planets in the system.

\section{HD 177830 (HIP 93746) \\ 5.1. Stellar properties}

HD 177830 is a $\mathrm{V}=7.177$ magnitude star of spectral class K0IV. Relative to the Sun, HD 177830 is quite metal-rich $([\mathrm{Fe} / \mathrm{H}]=0.55)$.

HD 177830 is a subgiant with $M_{v}=3.32$, close to giant status. The star has a known early $\mathrm{M}$ stellar companion with a projected separation of $97 \mathrm{AU}$ (Eggenberger et al. 2007). It was first reported by Vogt et al. (2000) to host a 392-day Jovian-mass planet in an eccentric $(e=0.42)$ orbit. Updates to the orbit were provided by Butler et al. (2006). Wright et al. (2007) noted a possible 111-day or 46.8-day signal but suggested that it could be correlated 
TABLE 4

KECK RADIAL VELOCITIES FOR HD 218566 (Sample: full table in electronic version)

\begin{tabular}{ccc}
\hline \hline Barycentric JD & RV $\left[\mathrm{m} \mathrm{s}^{-1}\right]$ & Uncertainty $\left[\mathrm{m} \mathrm{s}^{-1}\right]$ \\
\hline 2450366.85 & 4.96 & 1.16 \\
2450666.09 & -5.59 & 1.20 \\
2450690.03 & -5.47 & 1.27 \\
2450714.99 & 2.14 & 1.22 \\
2450715.94 & 3.39 & 1.16 \\
2450983.10 & 2.63 & 1.14 \\
2451012.04 & 5.34 & 1.35 \\
2451050.94 & 1.41 & 1.24 \\
2451071.96 & -1.88 & 1.19 \\
2451343.04 & -10.88 & 1.26 \\
2451369.04 & -9.64 & 1.23 \\
2451410.99 & -1.87 & 1.24 \\
2451440.89 & -3.74 & 1.36 \\
2451552.73 & -4.50 & 1.49 \\
2451900.74 & 4.46 & 1.17 \\
2452096.07 & -0.44 & 1.28 \\
2452242.73 & -5.51 & 1.47 \\
2452488.05 & -4.53 & 1.57 \\
2452535.87 & 1.94 & 1.27 \\
2452575.74 & 1.66 & 1.63 \\
& & \\
\hline
\end{tabular}

noise. Tanner et al. (2009) studied the star using Spitzer to place limits on the amount of dust in the system, and concluded that no significant excess emission at $160 \mu \mathrm{m}$ was detected (see also Trilling et al. 2008; Bryden et al. 2009).

The stellar parameters for this star listed in Table 1 are a compilation of various results, mostly from the SPOCS database Valenti \& Fischer (2005) with additions from the NStED database. The values for the stellar mass in Table 1 are the lower and upper limits of the isochrone mass listed in the SPOCS database. We find a current $\log R_{h k}^{\prime}$ value of -5.37. HD 177830 has a derived rotation period of 65 days (Barnes 2001).

\subsection{Keplerian solution}

We show the 88 Keck radial velocity measurements in Table 5. spanning approximately 15 years of RV monitoring. The median internal uncertainty for our observations is $1.05 \mathrm{~m} \mathrm{~s}^{-1}$, and the peak-to-peak velocity variation is $87.15 \mathrm{~m} \mathrm{~s}^{-1}$. The velocity scatter around the average RV in our observations is $24.68 \mathrm{~m} \mathrm{~s}^{-1}$.

The individual RV observations for HD 177830 are shown in the top panel of Figure 5. The middle panel shows the error-weighted Lomb-Scargle (LS) periodogram of the full RV dataset. Finally, the lower panel of Figure 5 shows the spectral window. The strongest peak in the periodogram is well-fit with a Keplerian model with period 407.31 days, semi-amplitude $K=31.17 \mathrm{~m} \mathrm{~s}^{-1}$ and estimated FAP $<3 \times 10^{-6}$. Together with the assumed stellar mass of $1.48 \mathcal{M}_{\odot}$, this amplitude corresponds to a minimum mass of $\mathcal{M} \sin i=$ $1.48 \mathcal{M}_{J}$. The best-fit orbit for the planet is essentially circular. This 1-planet fit achieves a reduced $\chi^{2}=27.53$, with an RMS of $5.24 \mathrm{~m} \mathrm{~s}^{-1}$. The top panel of Figure 6 shows the phased Keplerian fit for the 407-d planet.

The bottom panel of Figure 6 shows the periodogram of the residuals to the single-planet fit and the corresponding FAPs. The dominant peak at $\mathrm{P}=110.98$ with a FAP $\approx 5 \times 10^{-5}$ indicates the rather secure presence of an additional planet. Our best combined 2-planet fit indicates
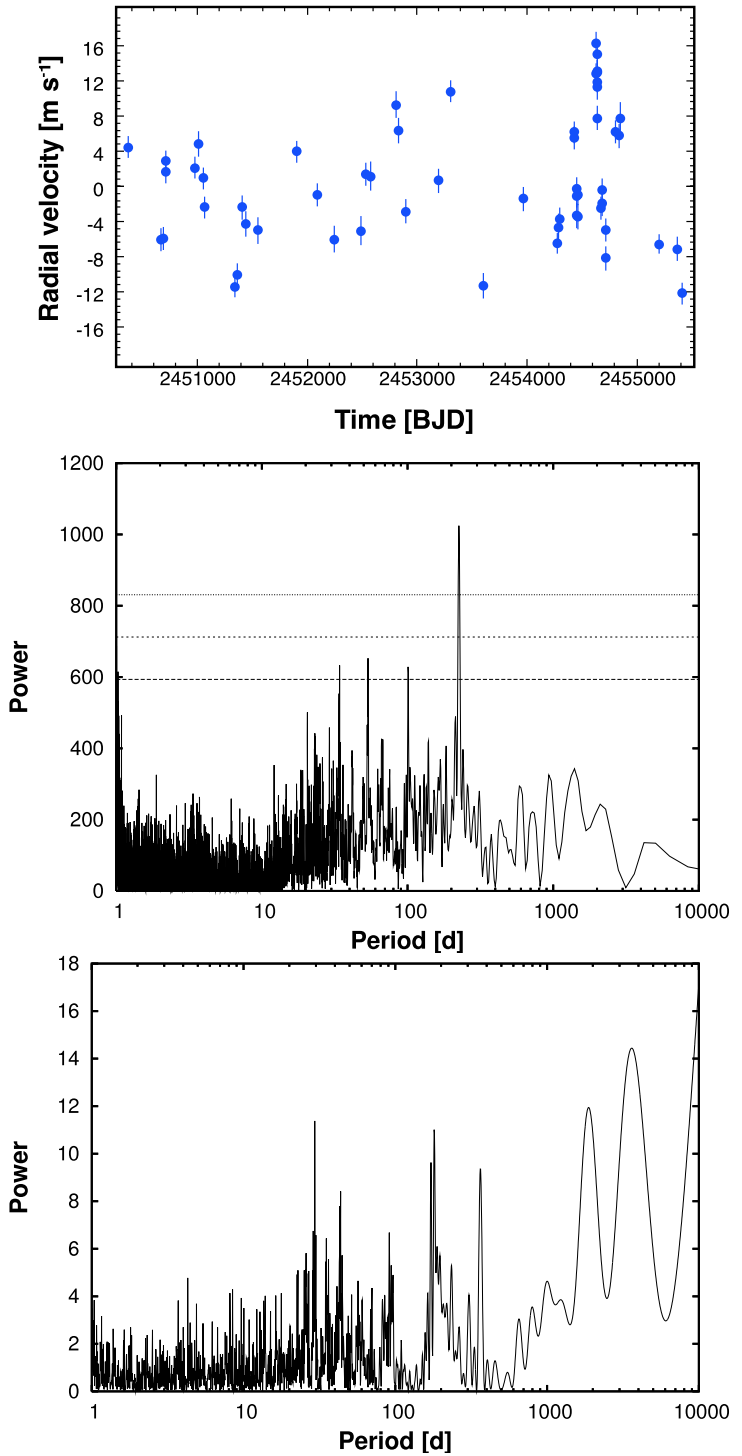

FIG. 3.- Radial velocity data and periodograms for HD 218566. Top panel: Relative radial velocity data obtained by KECK. Middle panel: Error-weighted Lomb-Scargle periodogram of the radial velocity data. Bottom panel: Power spectral window.

a new planet with $P=110.91$ days, $K=5.11 \mathrm{~m} \mathrm{~s}^{-1}$ and a minimum mass of $\mathcal{M} \sin i=0.15 \mathcal{M}_{J}$. The orbit of the second planet is moderately eccentric $(e \approx 0.36)$. With this revised fit, we obtain a reduced $\chi^{2}=15.31$ and an RMS of the residuals of approximately $3.85 \mathrm{~m} \mathrm{~s}^{-1}$. The expected jitter of HD 177830 (that is, the amount of jitter required to bring the reduced $\chi^{2}$ of the best-fit solution to 1.0$)$ is $3.71 \mathrm{~m} \mathrm{~s}^{-1}$.

The top and 2nd panels of Figure 7 show the phased stellar reflex velocity of HD 177830 due to each companion as compared to the RV dataset. The 3rd panel shows the residuals to the 2-planet solution, while the bottom panel shows the periodogram of the residuals of the best-fit solution. No compelling peaks are evident in the current Keck dataset, indicating that the present data offers no strong support for additional planets in the system.

The 2-planet fit shows a very slight amount of dynamical interaction between planets $\mathrm{b}$ and $\mathrm{c}$, which 

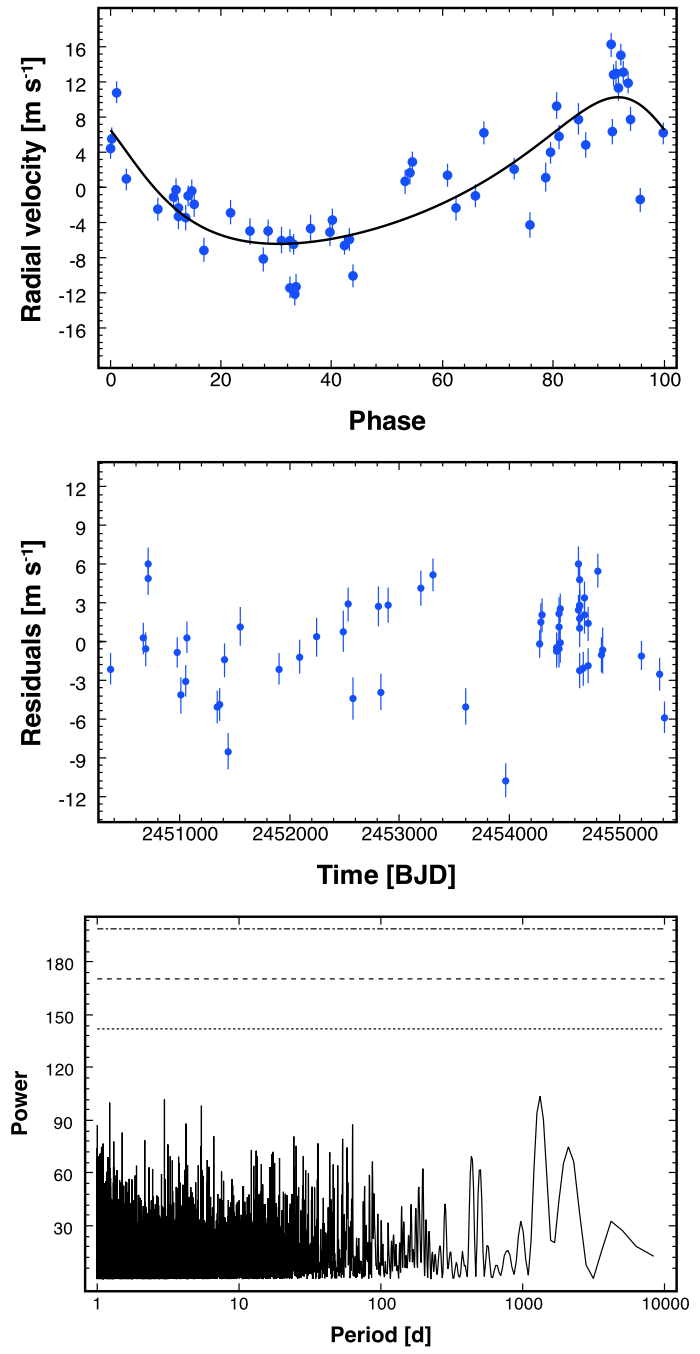

FIG. 4.- Keplerian solution and residuals periodogram for HD 218566. Top panel: Phased Keplerian fit. Middle panel: Residuals to the 1-planet Keplerian fit. Bottom panel: Periodogram of the residuals to the 1-planet best-fit solution.

we accounted for in the modeling using the BulirschStoer integration scheme in the Systemic console (Meschiari \& Laughlin 2010); we verified that the bestfit orbital model is stable for at least $10^{6}$ years. The time evolution of the eccentricity is shown in Figure 8

\section{HD 99492 (HIP 55848) \\ 6.1. Stellar properties}

HD 99492 is a $\mathrm{V}=7.383$ magnitude star of spectral type $\mathrm{K} 2 \mathrm{~V}$. A recent determination of many of its fundamental stellar parameters was given by Marcy et al. (2005) and is included in Table 1, supplemented with additional values from the NStED database. Marcy et al. (2005) found this star to be a middle-aged star of average chromospheric activity, with an age of 2-6 Gyr. They report an implied stellar rotation period of about 45 days $( \pm 30 \%)$ based on the star's chromospheric activity index. Marcy et al. (2005) also reported a 17.1-day $36 \mathcal{M}_{\oplus}$ planet orbiting this star. Compared to the Sun, HD 99492 is quite metal-rich $([\mathrm{Fe} / \mathrm{H}]=0.36)$.

\subsection{Keplerian solution}

TABLE 5

KECK RADIAL VElOCitiES FOR HD 177830 (Sample: full table in electronic version)

\begin{tabular}{ccc}
\hline \hline Barycentric JD & RV $\left[\mathrm{m} \mathrm{s}^{-1}\right]$ & Uncertainty $\left[\mathrm{m} \mathrm{s}^{-1}\right]$ \\
\hline 2450276.03 & -16.32 & 0.94 \\
2450605.04 & -5.29 & 0.95 \\
2450666.89 & -17.61 & 1.03 \\
2450982.94 & 0.32 & 1.11 \\
2451009.93 & -9.34 & 1.04 \\
2451068.82 & -30.65 & 0.95 \\
2451069.85 & -30.62 & 1.05 \\
2451070.90 & -28.30 & 1.00 \\
2451071.83 & -31.40 & 1.01 \\
2451072.82 & -27.97 & 1.00 \\
2451073.82 & -25.70 & 0.89 \\
2451074.81 & -33.00 & 0.98 \\
2451075.90 & -36.45 & 1.08 \\
2451311.11 & 30.25 & 1.07 \\
2451312.11 & 29.00 & 1.17 \\
2451313.11 & 18.91 & 1.02 \\
2451314.13 & 27.42 & 1.09 \\
2451341.95 & 27.81 & 0.96 \\
2451367.91 & 15.43 & 1.03 \\
2451368.91 & 11.00 & 1.17 \\
& &
\end{tabular}

Table 6 shows the 93 relative radial velocity measurements for HD 99492. The radial velocity coverage spans almost 14 years of RV monitoring. The median internal uncertainty for our observations is $1.36 \mathrm{~m} \mathrm{~s}^{-1}$, and the peak-to-peak velocity variation is $28.32 \mathrm{~m} \mathrm{~s}^{-1}$. The velocity scatter around the average RV in our observations is $6.39 \mathrm{~m} \mathrm{~s}^{-1}$.

The top panel of Figure 9 shows the individual RV observations for HD 99492. The middle panel shows the error-weighted Lomb-Scargle (LS) periodogram of the full RV dataset, while the bottom panel shows the spectral window. The FAP calculation for the strong Keplerian signal at $P=17.06$ days in the RV dataset indicates an estimated FAP $<3 \times 10^{-6}$. The dominant peak in the periodogram is well-fit by a Keplerian fit of period 17.05 days and semi-amplitude $K=7.86 \mathrm{~m} \mathrm{~s}^{-1}$. Together with the assumed stellar mass of $0.83 \mathcal{M}_{\odot}$, this amplitude suggests a minimum mass of $\mathcal{M} \sin i=27.76 \mathcal{M}_{\oplus}$. The best-fit orbit for the planet shows a small amount of eccentricity $(e \approx 0.13)$. This 1 -planet fit achieves a reduced $\chi^{2}=12.71$, with an RMS of $4.39 \mathrm{~m} \mathrm{~s}^{-1}$. The top panel of Figure 10] shows the phased Keplerian fit for the 17-d planet, while the bottom panel shows the periodogram of the residuals to the single-planet fit and the corresponding FAPs.

The additional peak in the periodogram of residuals with $\mathrm{P}=4908.67$ reveals the secure detection of an additional planet, with a FAP $\approx 4 \times 10^{-4}$. Our best combined 2-planet fit suggests a new planet with $P=4969.73$ days, $K=4.88 \mathrm{~m} \mathrm{~s}^{-1}$ and a minimum mass of $\mathcal{M} \sin i=0.36 \mathcal{M}_{J}$; the orbit of the second planet is somewhat eccentric $(e \approx 0.11)$. Using this revised fit, we obtain a reduced $\chi^{2}=7.17$ and an RMS of the residuals of approximately $3.22 \mathrm{~m} \mathrm{~s}^{-1}$. The expected jitter of HD 99492 (that is, the amount of jitter required to bring the reduced $\chi^{2}$ of the best-fit solution to 1.0$)$ is $2.94 \mathrm{~m} \mathrm{~s}^{-1}$.

The top and 2nd panels of Figure 11] show the phased stellar reflex velocity of HD 99492 from each planet compared to the RV dataset. The 3rd panel shows the residuals to the 2-planet solution, while the bottom panel 

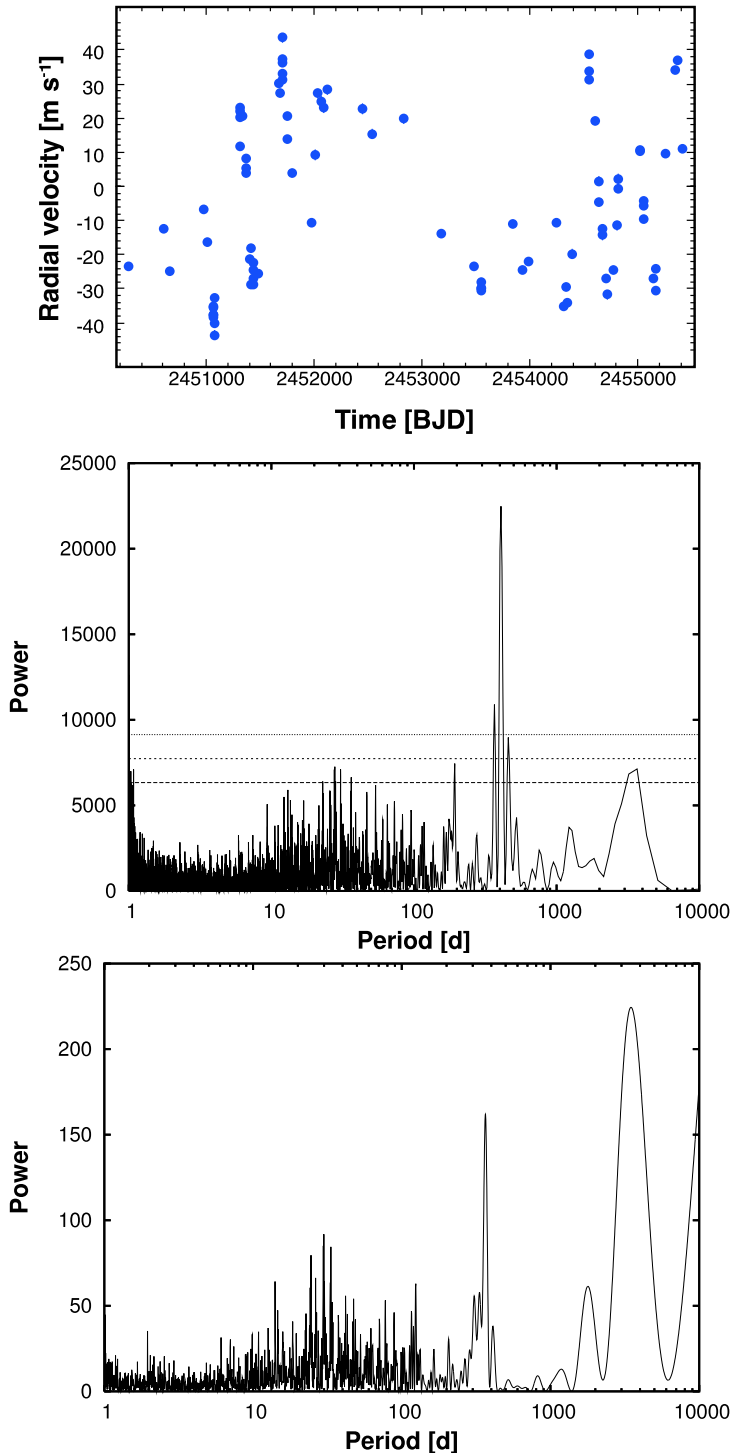

FIG. 5.- Radial velocity data and periodograms for HD 177830 Top panel: Relative radial velocity data obtained by KECK. Middle panel: Error-weighted Lomb-Scargle periodogram of the radial velocity data. Bottom panel: Power spectral window.

shows the periodogram of the residuals of the best-fit solution. No significant peaks are evident, indicating that the present data set offers no strong support for additional planets in the system.

\section{HD 74156 (HIP 42723)}

\subsection{Stellar properties}

HD 74156 is a $\mathrm{V}=7.614$ magnitude star of spectral type G1V. In comparison to the Sun, HD 74156 is modestly metal-rich $([\mathrm{Fe} / \mathrm{H}]=0.13)$.

HD 74156 is a well-studied star, known already to have both a 52-day and a 2500-day planet (Naef et al. 2004). The star was claimed by Bean et al. (2008) to also harbor a 3rd planet ("d") at 336 days, in apparent support of the so-called "Packed Planetary Systems" hypothesis (PPS; Barnes \& Raymond 2004). Indeed, Barnes \& Greenberg (2007) cited the discovery of d as a successful prediction of the PPS hypothesis. However, the reality of HD 74516 d was called into question by Baluev (2009) as a false detection made due to annual systematic errors in
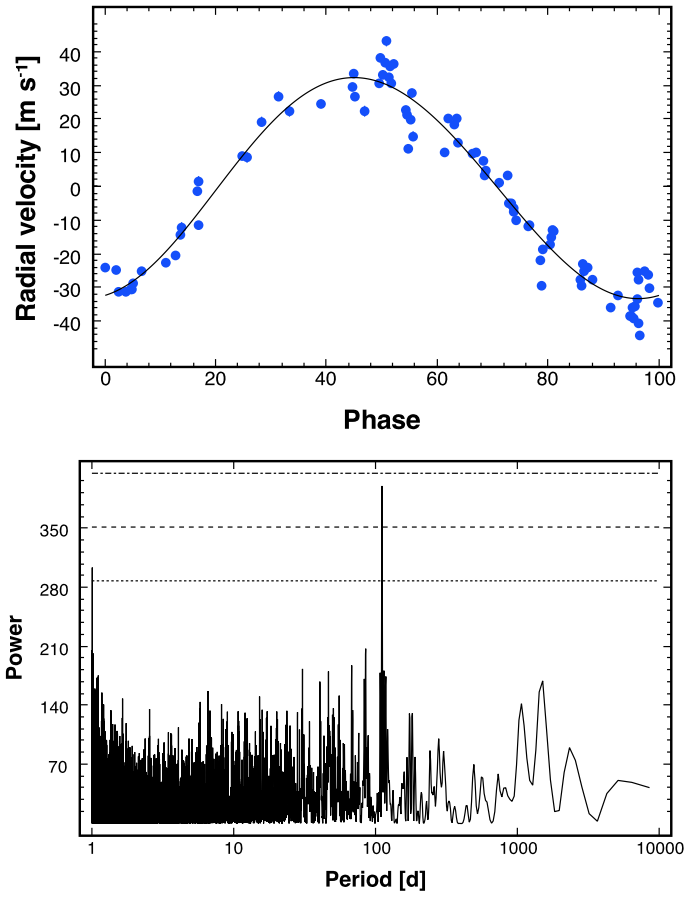

FIG. 6.- One-planet Keplerian solution and residuals periodogram for HD 177830. Top panel: Phased Keplerian fit. Bottom panel: Periodogram of the residuals to the 1-planet best-fit solution.

TABLE 6

KECK RADIAL VELOCITIES FOR HD 99492 (Sample: full table in electronic version)

\begin{tabular}{ccc}
\hline \hline Barycentric JD & RV $\left[\mathrm{m} \mathrm{s}^{-1}\right]$ & Uncertainty $\left[\mathrm{m} \mathrm{s}^{-1}\right]$ \\
\hline 2450462.11 & -2.62 & 1.51 \\
2450546.99 & -3.46 & 1.39 \\
2450837.93 & -2.66 & 1.58 \\
2450862.90 & -4.70 & 1.51 \\
2450955.88 & -7.05 & 1.18 \\
2451172.10 & -4.12 & 1.59 \\
2451228.04 & -6.80 & 1.53 \\
2451311.82 & 3.26 & 1.63 \\
2451544.17 & -6.23 & 1.27 \\
2451582.97 & 0.43 & 1.34 \\
2451704.81 & -1.45 & 1.60 \\
2451898.15 & -14.46 & 1.41 \\
2451973.05 & 3.64 & 1.37 \\
2452095.75 & -0.65 & 1.55 \\
2452097.75 & -7.23 & 1.56 \\
2452333.14 & 6.24 & 1.65 \\
2452334.08 & 0.68 & 1.62 \\
2452334.97 & 1.21 & 1.59 \\
2452364.07 & 3.76 & 1.44 \\
2452445.77 & -6.52 & 1.50 \\
& & \\
\hline
\end{tabular}

the HET RV data. Wittenmyer et al. (2009) also found no evidence of HD $74156 \mathrm{~d}$ in their follow-up study.

We have had HD 74156 under precise radial velocity monitoring at Keck for the past 8.9 years and here add 21 new velocities to the mix, combined with previously published data from CORALIE, ELODIE, and HET, bringing the total number of observations to 198 . We re-analyzed the compound dataset from scratch, looking for evidence of further planetary companions. As usual, we allowed a floating offset between each data set in the Keplerian fitting process to compensate for the different 

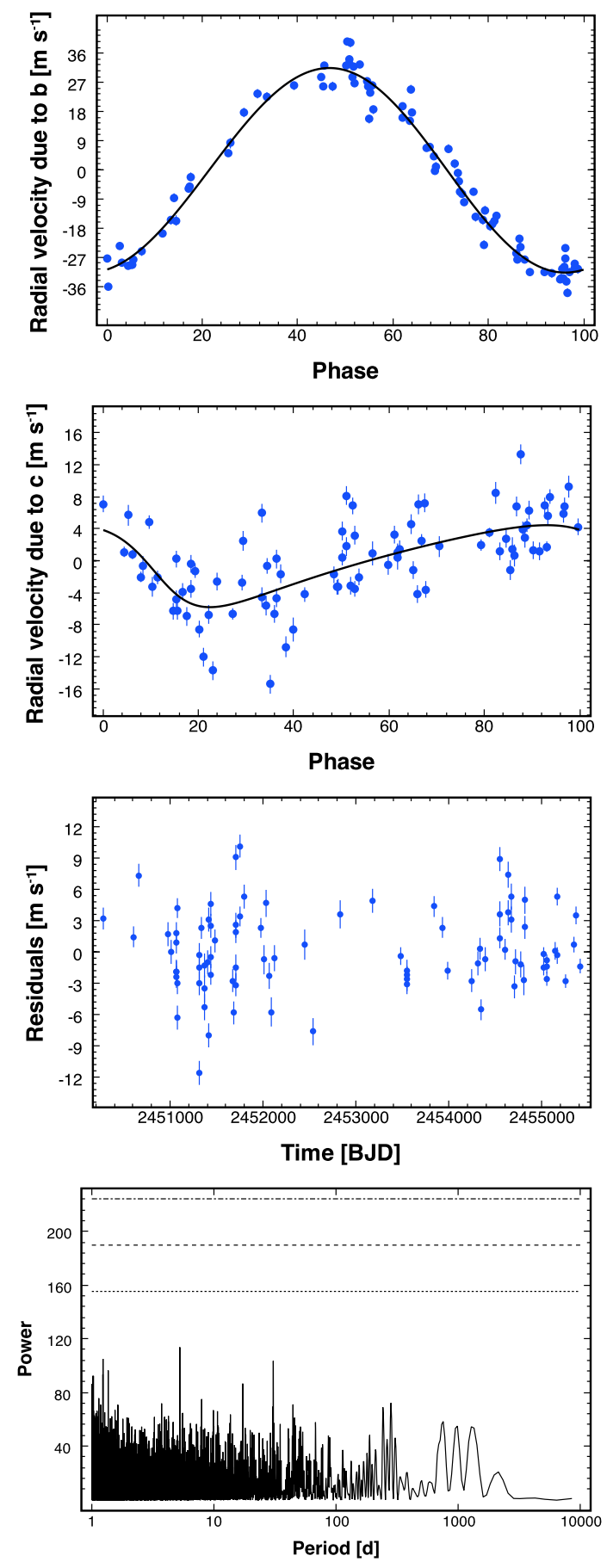

FIG. 7.- Keplerian solution and residuals periodogram for HD 177830. 1st panel: Phased Keplerian fit of the 407-d component b. 2nd panel: Phased Keplerian fit of the 111-d component c. 3rd panel: Residuals to the 2-planet fit. 4th panel: Periodogram of the residuals to the 2-planet best fit solution.

zero-points of each observatory.

\subsection{Keplerian solution}

Table[7shows Keck/HIRES relative radial velocity observations for HD 74156. The radial velocity coverage spans almost 13 years of RV monitoring. The top panel of Figure 12 shows the individual RV observations for HD 74156 (CORALIE04, ELODIE04 Naef et al. (2004), HET09 Wittenmyer et al. (2009) and KECK; each RV

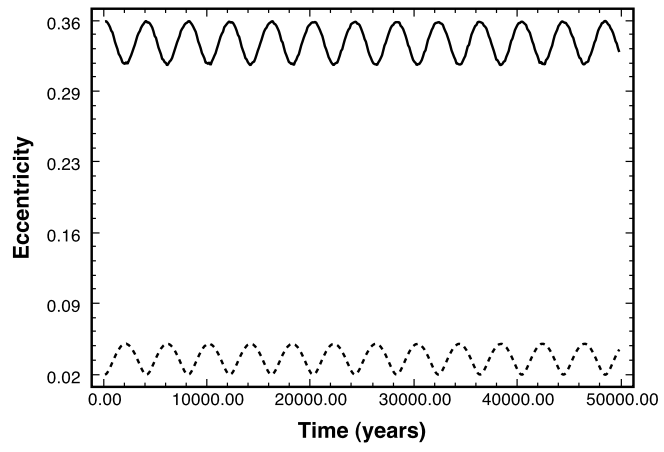

FIG. 8. - Eccentricity evolution of planets HD177830 b (dashed line) and c (solid line) within $5 \times 10^{4}$ years.
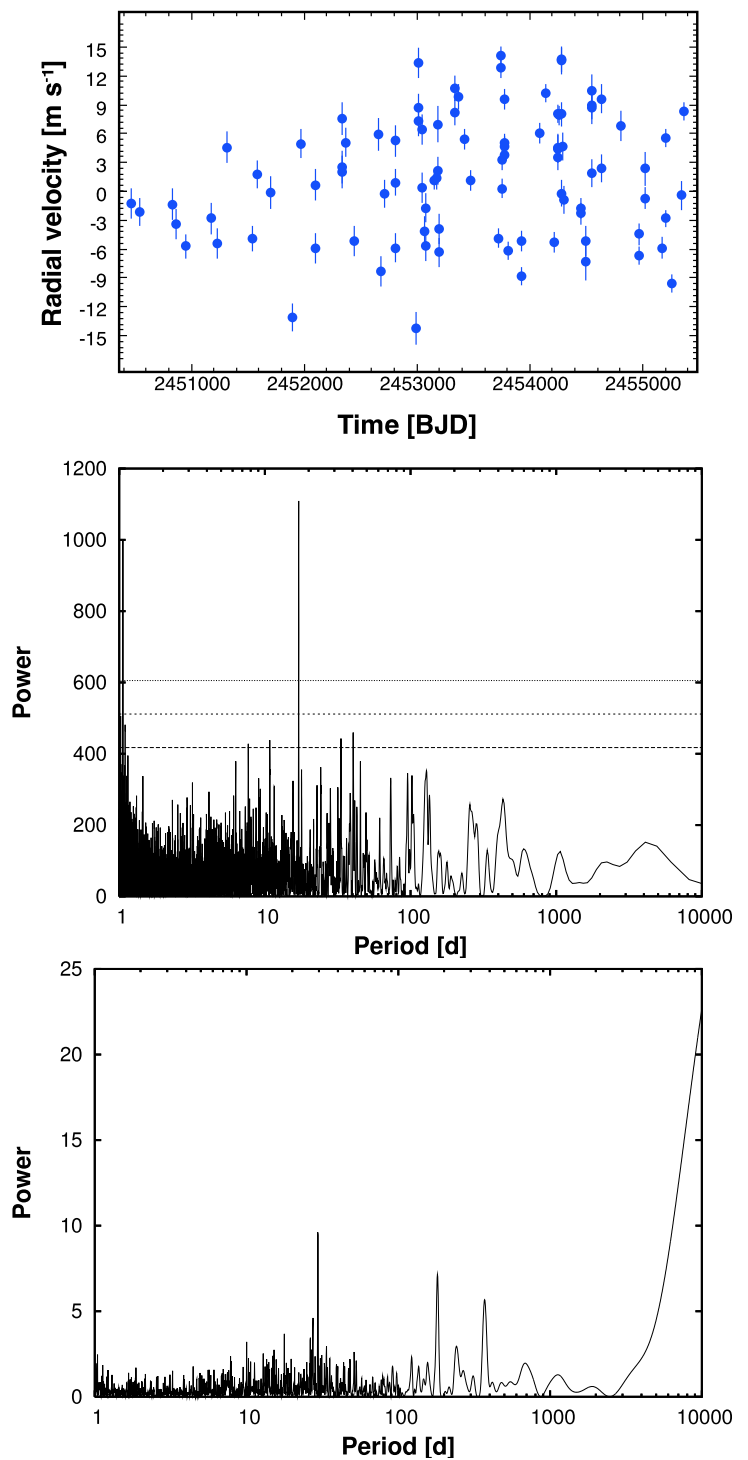

FIG. 9.- Radial velocity data and periodograms for HD 99492. Top panel: Relative radial velocity data obtained by KECK. Middle panel: Error-weighted Lomb-Scargle periodogram of the radial velocity data. Bottom panel: Power spectral window. 

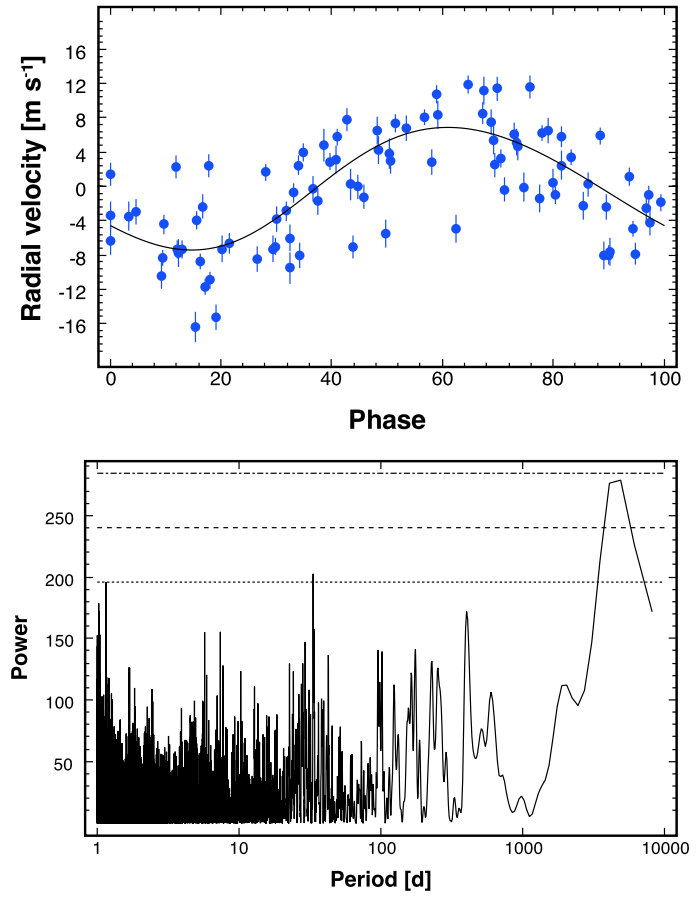

FIG. 10.- One-planet Keplerian solution and residuals periodogram for HD 99492. Top panel: Phased Keplerian fit. Bottom panel: Periodogram of the residuals to the 1-planet best-fit solution.

dataset has been offset to yield the best-fit solution). The middle panel shows the error-weighted Lomb-Scargle (LS) periodogram of the full RV dataset. Figures 13 and 14 show the best 1-planet and 2-planet fits, respectively. The best 2-planet fit (derived using the full set of RV observations) obtains a reduced $\chi^{2}=3.09$, an RMS of the residuals of approximately $12.80 \mathrm{~m} \mathrm{~s}^{-1}$ and an expected jitter of $8.59 \mathrm{~m} \mathrm{~s}^{-1}$. The value of the estimated jitter from this best-fit is considerably higher than the $2.2 \mathrm{~m}$ $\mathrm{s}^{-1}$ expected from its $\log R_{h k}^{\prime}$ activity index. However, in this case the RMS is dominated by the CORALIE and ELODIE data, with a considerable contribution also from the HET data. The RMS of the fit using only the 29 Keck points is $3.5 \mathrm{~m} \mathrm{~s}^{-1}$ with jitter of $2.9 \mathrm{~m} \mathrm{~s}^{-1}$, in much closer accord with the expected stellar jitter of 2.2 $\mathrm{m} \mathrm{s}^{-1}$.

The periodogram shown in the bottom panel of Figure 14. shows no compelling peaks in the residuals, indicating that the present data set offers no significant support for additional planets in the system. Our results confirm the conclusions of Wittenmyer et al. (2009). The expanded dataset presented in this paper does not support the theoretical and observational evidence for a third planetary companion claimed by Bean et al. (2008).

\section{CONCLUSIONS}

The five systems presented in this paper add to the ever-growing list of single and multiple-components exoplanetary systems.

Two of the systems, namely, HD 31253 and HD 218566 , are well-characterized Saturn-mass planets in $\approx 1$-year orbits. For both systems, the data presented in this paper do not show prominent peaks in the periodogram of residuals, even at a 10\% FAP level. While several single-planet systems with similar properties have later
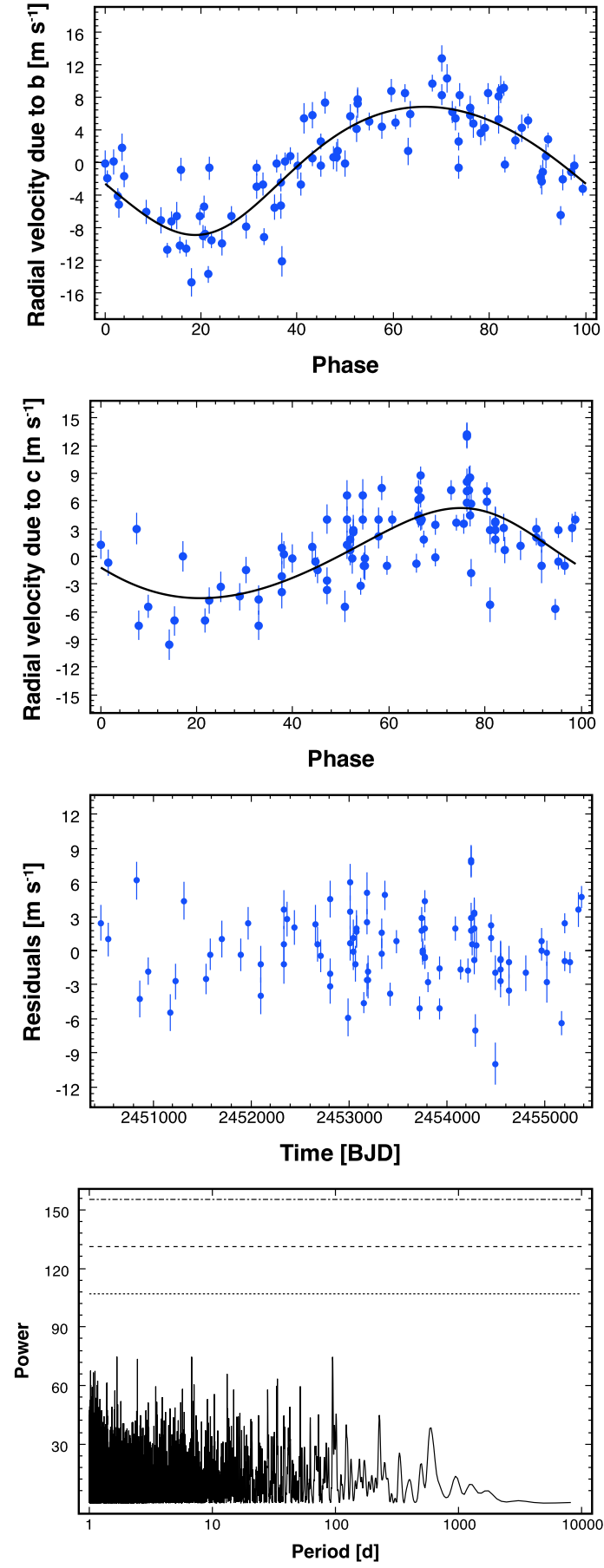

FIG. 11.- Keplerian solution and residuals periodogram for HD 99492. 1st panel: Phased Keplerian fit of the 17-d component b. 2nd panel: Phased Keplerian fit of the 4697-d component c. 3rd panel: Residuals to the 2-planet fit. 4th panel: Periodogram of the residuals to the 2-planet best fit solution.

been characterized with an additional outer, long-period companion (e.g. Jones et al. 2010), the absence of any significant linear trend in the current data seem to rule out the presence of additional Jupiter-mass planets with $P<40,000$ days.

HD 99492 and HD 177830 each gain a new planetary companion, adding to the previously known planets (Vogt et al. 2000; Marcy et al. 2005). The known linear trend in the residuals to HD 99492 b is now fully char- 
TABLE 7

KECK RADIAL VELOCITIES FOR HD 74156 (Sample: full table in electronic version)

\begin{tabular}{ccc}
\hline \hline Barycentric JD & RV $\left[\mathrm{m} \mathrm{s}^{-1}\right]$ & Uncertainty $\left[\mathrm{m} \mathrm{s}^{-1}\right]$ \\
\hline 2452007.90 & 99.69 & 1.97 \\
2452236.01 & -35.73 & 2.08 \\
2452243.12 & -35.62 & 1.89 \\
2452307.89 & 35.08 & 2.32 \\
2452573.14 & 14.26 & 2.12 \\
2452682.96 & 0.00 & 2.31 \\
2452711.82 & -43.62 & 2.32 \\
2452777.80 & -18.09 & 1.76 \\
2453017.86 & -135.63 & 2.06 \\
2453339.08 & -3.45 & 1.83 \\
2453426.89 & -169.07 & 1.87 \\
2453746.94 & 153.42 & 1.91 \\
2454428.07 & 117.86 & 1.83 \\
2454461.07 & -68.78 & 2.26 \\
2454464.99 & 32.97 & 2.02 \\
2454490.94 & 106.20 & 1.98 \\
2454492.91 & 105.85 & 1.93 \\
2454545.89 & 94.84 & 2.01 \\
2454601.83 & 77.13 & 1.95 \\
2455202.89 & -1.01 & 1.41 \\
& & \\
\hline
\end{tabular}

acterized thanks to the longer phase coverage, indicating the presence of a Saturn-mass planet on a $\sim 5,000$-day orbit.

The RV data we collected for HD 177830 support the existence of an additional inner planet, presenting an interesting case. The planets in this system are within a binary with a separation of approximately 97 AU (Eggenberger et al. 2007). Simulations of the formation and stability of planets in binary star systems imply that the perturbative effect of the secondary star will be negligible in binaries with separation larger than $100 \mathrm{AU}$. The binary system of HD 177830 is slightly below this limit. This system is also the first binary with a moderate separation in which multiple planets have been discovered. Although it is unlikely that the low-mass secondary star of this system has had significant effects on the formation of planets around the primary, it would still be interesting to study how planets in this system formed and migrated to their current stable orbits.

Finally, we analyzed an expanded dataset of Doppler observations of HD 74156, adding 21 Keck RV points to the known data. We repeated the analysis looking for evidence of a third planet, which would lend observational credence to the predictions of the PPS hypothesis (e.g. Barnes \& Ravmond 2004). Our dataset does not show support for the claimed HD 74156 d planetary companion. Indeed, the residuals periodogram to our best 2-planet fit do not exhibit any promising peaks for future RV follow-ups, strengthening the conclusions of Wittenmver et al. (2009).

All the planets presented in this paper lie well within the existing exoplanet parameter envelopes (Fig. [15). Several of them lie in the so-called "desert" in the mass and semi-major axis distribution of extrasolar planets (Ida \& Lin 2004). Monte-Carlo population synthesis models for extrasolar giant planet formation tend to suggest that planets migrate relatively rapidly through the period range between 10 and 100 days, and, in addition, often grow quickly through the mass range centered on the Saturnian mass. In the context of the overall plane-
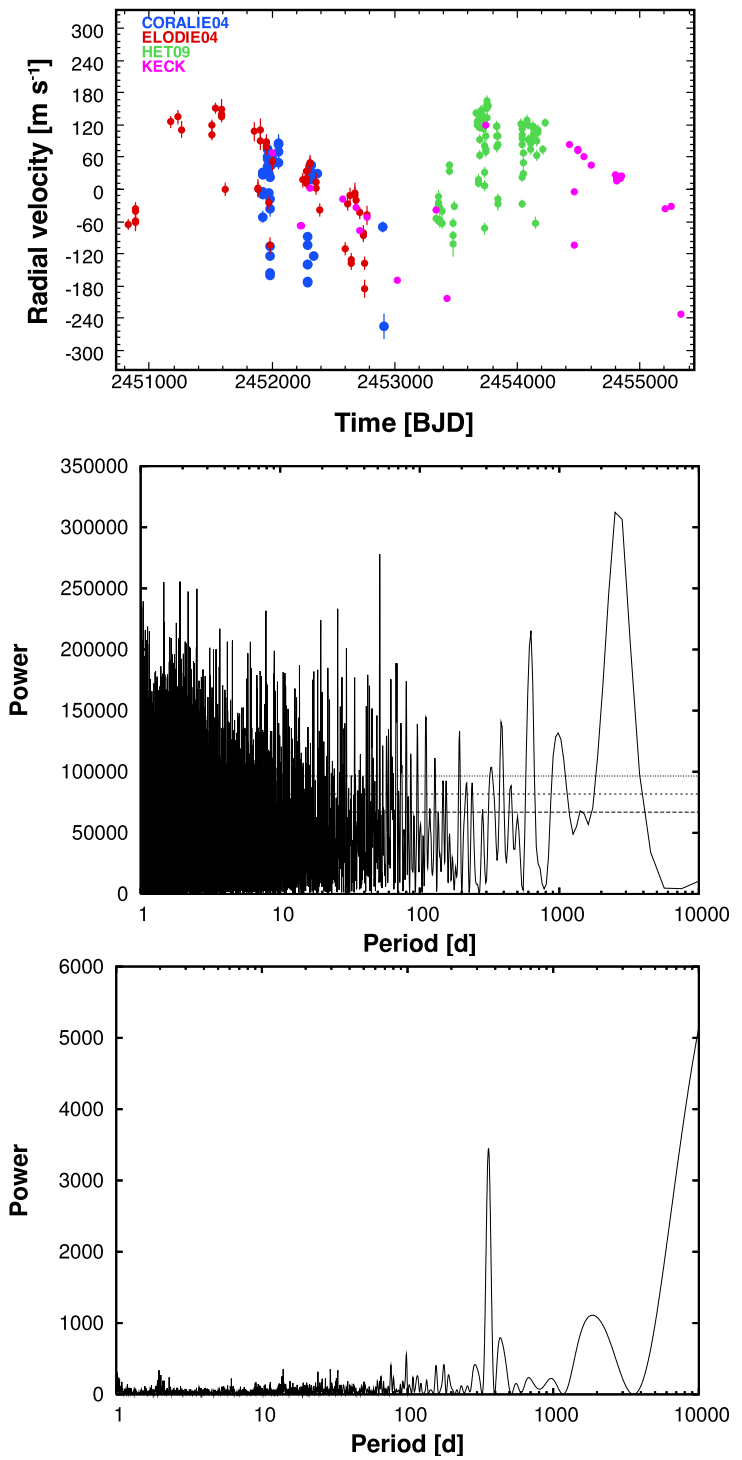

FIG. 12.- Radial velocity data and periodograms for HD 74156. Top panel: Relative radial velocity data obtained by CORALIE04, ELODIE04 (Naef et al. 2004), HET09 (Wittenmver et al. 2009) and KECK. Middle panel: Error-weighted Lomb-Scargle periodogram of the radial velocity data. Bottom panel: Power spectral window.

tary census, these four new planets help to further elucidate the various statistical properties of exoplanets. In particular, the discovery of multiple-planet systems helps in further characterizing the number of stars hosting multiple planetary companions and any correlations emerging in the distribution of orbital elements as suggested by observational clues (e.g. Wright et al. 2009). 

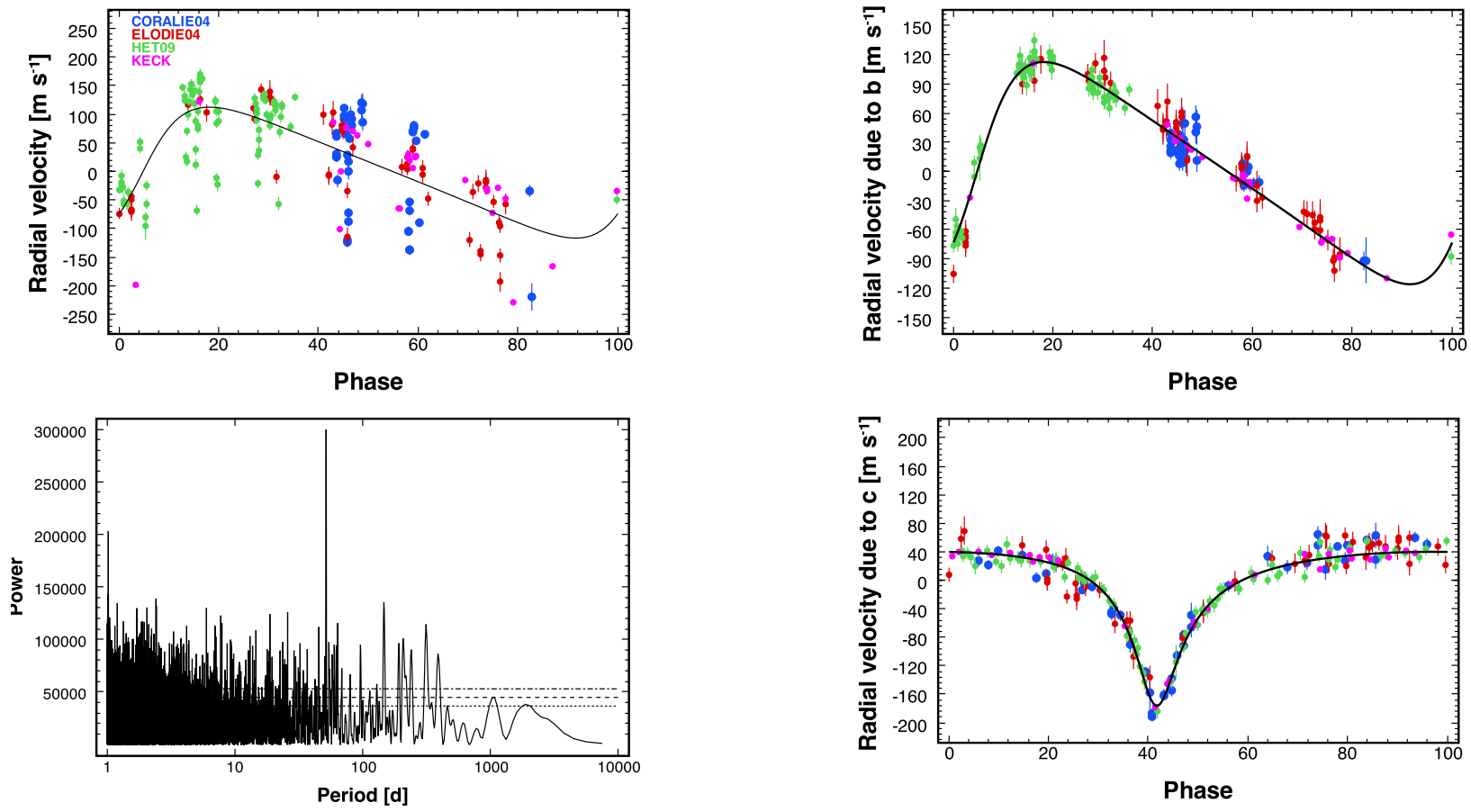

FIG. 13.- One-planet Keplerian solution and residuals periodogram for HD 74156. Top panel: Phased Keplerian fit. Bottom panel: Periodogram of the residuals to the 1-planet best-fit solution.
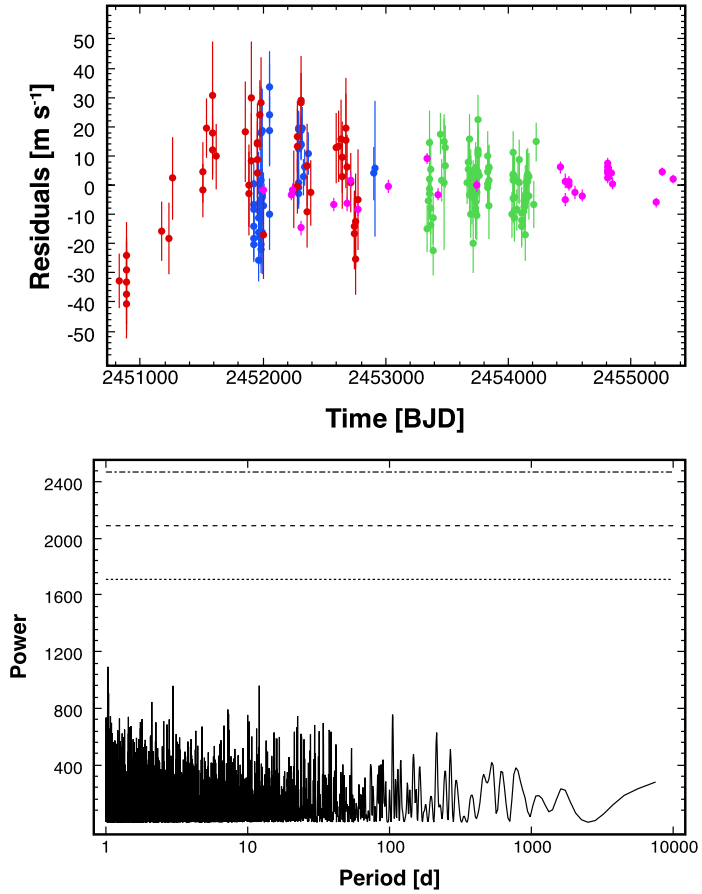

FIG. 14.- Keplerian solution and residuals periodogram for HD 74156. 1st panel: Phased Keplerian fit of the 52-d component b. 2nd panel: Phased Keplerian fit of the 2514-d component c. 3rd panel: Residuals to the 2-planet fit. 4th panel: Periodogram of the residuals to the 2-planet best fit solution. 

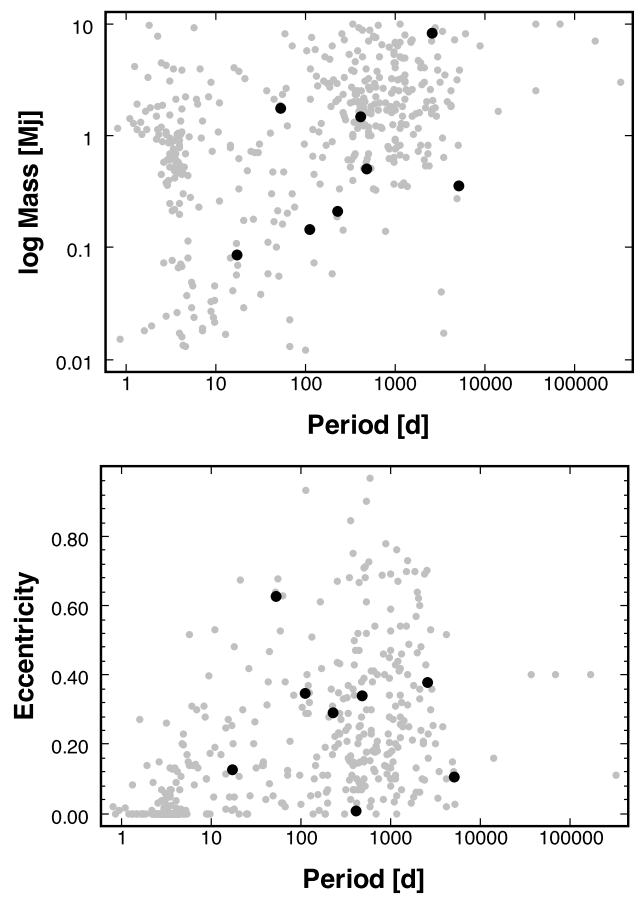

FIG. 15.- Plot of all known extrasolar planets (gray dots) and the orbital elements of all the planets orbiting the host stars presented in this paper (black points). 
G.L. acknowledges support from the NASA Astrobiology Institute at NASA Ames Research Center, and from the NSF CAREER Program through NSF Grant AST0449986. S.S.V gratefully acknowledges support from NSF grants AST-0307493 and AST-0908870. R.P.B. gratefully acknowledges support from NASA OSS Grant NNX07AR40G, the NASA Keck PI program, and from the Carnegie Institution of Washington. N.H. acknowledges support from the NASA Astrobiology Institute under Cooperative Agreement NNA04CC08A at the Institute for Astronomy, University of Hawaii, and the NASA/EXOB grant NNX09AN05G. The work herein is based on observations obtained at the W. M. Keck Ob- servatory, which is operated jointly by the University of California and the California Institute of Technology, and we thank the UC-Keck, UH-Keck and NASA-Keck Time Assignment Committees for their support. We also wish to extend our special thanks to those of Hawaiian ancestry on whose sacred mountain of Mauna Kea we are privileged to be guests. Without their generous hospitality, the Keck observations presented herein would not have been possible. This research has made use of the NStEd database operated by NASA and the SIMBAD database, operated at CDS, Strasbourg, France. This paper was produced using ${ }^{B} A^{M}$.

Facilities: Keck (HIRES)

\section{REFERENCES}

Baluev, R. V. 2009, MNRAS, 393, 969

Barnes, R., Goździewski, K., \& Raymond, S. N. 2008, ApJ, 680, L57

Barnes, R., \& Greenberg, R. 2007, ApJ, 665, L67

Barnes, R., \& Raymond, S. N. 2004, ApJ, 617, 569

Barnes, S. A. 2001, ApJ, 561, 1095

Bean, J. L., Benedict, G. F., Charbonneau, D., Homeier, D., Taylor, D. C., McArthur, B., Seifahrt, A., Dreizler, S., \& Reiners, A. 2008, A\&A, 486, 1039

Bean, J. L., \& Seifahrt, A. 2009, A\&A, 496, 249

Benedict, G. F., McArthur, B. E., Forveille, T., Delfosse, X., Nelan, E., Butler, R. P., Spiesman, W., Marcy, G., Goldman, B., Perrier, C., Jefferys, W. H., \& Mayor, M. 2002, ApJ, 581, L115

Bennett, D. P., et al. 2009, astro2010: The Astronomy and Astrophysics Decadal Survey, 2010, 18

Bryden, G., Beichman, C. A., Carpenter, J. M., Rieke, G. H., Stapelfeldt, K. R., Werner, M. W., Tanner, A. M., Lawler, S. M., Wyatt, M. C., Trilling, D. E., Su, K. Y. L., Blaylock, M., \& Stansberry, J. A. 2009, ApJ, 705, 1226

Butler, R. P., Marcy, G. W., Williams, E., McCarthy, C., Dosanjh, P., \& Vogt, S. S. 1996, PASP, 108, 500

Butler, R. P., Wright, J. T., Marcy, G. W., Fischer, D. A., Vogt, S. S., Tinney, C. G., Jones, H. R. A., Carter, B. D., Johnson, J. A., McCarthy, C., \& Penny, A. J. 2006, ApJ, 646, 505

Charbonneau, D., Brown, T. M., Burrows, A., \& Laughlin, G. 2007, in Protostars and Planets V, ed. B. Reipurth, D. Jewitt, \& K. Keil, 701-716

Charbonneau, D., Brown, T. M., Latham, D. W., \& Mayor, M. 2000, ApJ, 529, L45

Chauvin, G., Lagrange, A.-M., Zuckerman, B., Dumas, C., Mouillet, D., Song, I., Beuzit, J.-L., Lowrance, P., \& Bessell, M. S. 2005, A\&A, 438, L29

Dawson, R. I., \& Fabrycky, D. C. 2010, ApJ, 722, 937

Eggenberger, A., Udry, S., Chauvin, G., Beuzit, J., Lagrange, A., Ségransan, D., \& Mayor, M. 2007, A\&A, 474, 273

Gilliland, R. L., \& Baliunas, S. L. 1987, ApJ, 314, 766

Henry, G. W., Marcy, G. W., Butler, R. P., \& Vogt, S. S. 2000, ApJ, 529, L41

Ida, S., \& Lin, D. N. C. 2004, ApJ, 604, 388

Jones, H. R. A., Butler, R. P., Tinney, C. G., O'Toole, S., Wittenmyer, R., Henry, G. W., Meschiari, S., Vogt, S., Rivera, E., Laughlin, G., Carter, B. D., Bailey, J., \& Jenkins, J. S. 2010, MNRAS, 403, 1703

Kalas, P., Graham, J. R., Chiang, E., Fitzgerald, M. P., Clampin, M., Kite, E. S., Stapelfeldt, K., Marois, C., \& Krist, J. 2008, Science, 322, 1345

Marcy, G. W., Butler, R. P., Vogt, S. S., Fischer, D. A., Henry, G. W., Laughlin, G., Wright, J. T., \& Johnson, J. A. 2005, ApJ, 619, 570

Marois, C., Macintosh, B., Barman, T., Zuckerman, B., Song, I., Patience, J., Lafrenière, D., \& Doyon, R. 2008, Science, 322, 1348
Mayor, M., \& Queloz, D. 1995, Nature, 378, 355

Mayor, M., Udry, S., Lovis, C., Pepe, F., Queloz, D., Benz, W., Bertaux, J.-L., Bouchy, F., Mordasini, C., \& Segransan, D. 2009, A\&A, 493, 639

Meschiari, S., Wolf, A. S., Rivera, E., Laughlin, G., Vogt, S., \& Butler, P. 2009, PASP, 121, 1016

Meschiari, S., \& Laughlin, G. P. 2010, ApJ, 718, 543

Naef, D., Mayor, M., Beuzit, J. L., Perrier, C., Queloz, D., Sivan, J. P., \& Udry, S. 2004, A\&A, 414, 351

Nordström, B., Mayor, M., Andersen, J., Holmberg, J., Pont, F., Jørgensen, B. R., Olsen, E. H., Udry, S., \& Mowlavi, N. 2004, A\&A, 418, 989

Press, W. H., Teukolsky, S. A., Vetterling, W. T., \& Flannery, B. P. 1992, Numerical recipes in C. The art of scientific computing (Cambridge: University Press, — c1992, 2nd ed.)

Rivera, E. J., Lissauer, J. J., Butler, R. P., Marcy, G. W., Vogt, S. S., Fischer, D. A., Brown, T. M., Laughlin, G., \& Henry, G. W. 2005, ApJ, 634, 625

Silvotti, R., Schuh, S., Janulis, R., Solheim, J.-E., Bernabei, S., Østensen, R., Oswalt, T. D., Bruni, I., Gualandi, R., Bonanno, A., Vauclair, G., Reed, M., Chen, C.-W., Leibowitz, E., Paparo, M., Baran, A., Charpinet, S., Dolez, N., Kawaler, S., Kurtz, D., Moskalik, P., Riddle, R., \& Zola, S. 2007, Nature, 449, 189

Takeda, G., Ford, E. B., Sills, A., Rasio, F. A., Fischer, D. A., \& Valenti, J. A. 2007, ApJS, 168, 297

Tanner, A., Beichman, C., Bryden, G., Lisse, C., \& Lawler, S. 2009, ApJ, 704, 109

Trilling, D. E., Bryden, G., Beichman, C. A., Rieke, G. H., Su, K. Y. L., Stansberry, J. A., Blaylock, M., Stapelfeldt, K. R., Beeman, J. W., \& Haller, E. E. 2008, ApJ, 674, 1086

Udry, S., Fischer, D., \& Queloz, D. 2007, in Protostars and Planets V, ed. B. Reipurth, D. Jewitt, \& K. Keil, 685-699

Valenti, J. A., \& Fischer, D. A. 2005, ApJS, 159, 141

Vogt, S. S., Marcy, G. W., Butler, R. P., \& Apps, K. 2000, ApJ, 536, 902

Vogt, S. S., Wittenmyer, R. A., Butler, R. P., O'Toole, S., Henry, G. W., Rivera, E. J., Meschiari, S., Laughlin, G., Tinney, C. G., Jones, H. R. A., Bailey, J., Carter, B. D., \& Batygin, K. 2010, ApJ, 708, 1366

Vogt, S. S., et al. 1994, in Society of Photo-Optical

Instrumentation Engineers (SPIE) Conference Series, Vol. 2198, Society of Photo-Optical Instrumentation Engineers (SPIE) Conference Series, ed. D. L. Crawford \& E. R. Craine, 362-+

Wittenmyer, R. A., Endl, M., Cochran, W. D., Levison, H. F., \& Henry, G. W. 2009, ApJS, 182, 97

Wright, J. T. 2005, PASP, 117, 657

Wright, J. T., Marcy, G. W., Butler, R. P., \& Vogt, S. S. 2004 , ApJS, 152, 261

Wright, J. T., Marcy, G. W., Fischer, D. A., Butler, R. P., Vogt, S. S., Tinney, C. G., Jones, H. R. A., Carter, B. D., Johnson, J. A., McCarthy, C., \& Apps, K. 2007, ApJ, 657, 533

Wright, J. T., Upadhyay, S., Marcy, G. W., Fischer, D. A., Ford, E. B., \& Johnson, J. A. 2009, ApJ, 693, 1084 